\title{
Matrine increases the inhibitory effects of afatinib on H1975 cells via the IL-6/JAK1/STAT3 signaling pathway
}

\author{
SHUI-FANG CHEN, ZE-YING ZHANG and JIAN-LI ZHANG
}

\author{
Respiratory Department, The First Affiliated Hospital, College of Medicine, \\ Zhejiang University, Hangzhou, Zhejiang 310003, P.R. China
}

Received October 15, 2016; Accepted June 8, 2017

DOI: $10.3892 / \mathrm{mmr} .2017 .6865$

\begin{abstract}
Resistance to epidermal growth factor receptor (EGFR) inhibitors is of primary concern in the treatment of non-small-cell lung cancer (NSCLC) with EGFR mutations. To investigate the effects of matrine on H1975 cells and to examine a novel, potential treatment option for NSCLC, the present study measured cell viability, apoptotic rate, interleukin 6 (IL-6) expression and activation of the janus kinase (JAK) 1/signal transducer and activator of transcription (STAT)3 signaling pathway in cells treated with or without matrine, in the presence or absence of afatinib. The results demonstrated that matrine treatment inhibited cell growth, decreased B-cell lymphoma 2 (Bcl-2) expression and induced apoptosis. Matrine treatment additionally decreased the mRNA and protein levels of IL-6 and inhibited activation of the JAK1/STAT3 signaling pathway in H1975 cells in a dose-dependent manner. H1975 cells treated with IL-6 small interfering RNA exhibited a decrease in Bcl-2 expression levels and cell viability. Treatment with a combination of matrine and afatinib demonstrated increased inhibitory effects on the growth rate of H1975 cells. The findings of the present study suggested that matrine treatment decreases IL-6 expression, inhibits activation of the JAK1/STAT3 signaling pathway, reduces the expression levels of $\mathrm{Bcl}-2$ and inhibits cell growth. Furthermore, matrine treatment was demonstrated to increase the inhibitory effects of afatinib on H1975 cells with the T790M EGFR mutation.
\end{abstract}

\section{Introduction}

Non-small-cell lung cancer (NSCLC) represents approximately $80 \%$ of lung cancers, and is the leading cause of cancer death with 5 -year survival rates less than $15 \%(1,2)$.

Correspondence to: Dr Shui-Fang Chen, Respiratory Department, The First Affiliated Hospital, College of Medicine, Zhejiang University, 79 Qingchun Road, Hangzhou, Zhejiang 310003, P.R. China

E-mail: chensf@mail.hz.zj.cn

Key words: non-small-cell lung cancer (NSCLC), matrine, afatinib, IL-6/JAK1/STAT3 signaling pathway
The epidermal growth factor receptor (EGFR, also called HER1), a tumour-promoting factor (3), plays a considerable role in NSCLC, especially in cells with activating EGFR mutations, such as L858R and exon-19 deletion $(4,5)$. EGFR expression was considered as a predictor of survival for first-line chemotherapy plus cetuximab in patients with advanced NSCLC (6). EGFR activation can be inhibited with tyrosine kinase inhibitors (TKIs), including afatinib, gefitinib, and erlotinib, which disrupt oncogenic cell signaling pathways and benefit patients with sensitizing mutations. Approximately $70 \%$ of patients respond initially to TKI treatment but eventually develop resistance with a median time of 10-16 months (7). In at least $50 \%$ of these cases, the mutation T790M in exon 20 of EGFR arises (8). This mutation has been reported to reverse inactivation of the EGFR induced by TKIs by altering the structure of the EGFR such that the ability of the EGFR to bind TKIs is reduced (8). TKIs have also proved to induce expression of IL-6, which activates the JAK1/STAT3 signaling pathway through the IL-6 receptor (IL-6R), leading to increased survival (9-11). TKI-dependent expression of IL- 6 increases expression of the transforming growth factor $\beta 1$ (TGF- $\beta 1$ ) receptor and enhances TGF- $\beta 1$ signaling, contributing to the epithelial-mesenchymal transition (EMT) and tumor progression $(12,13)$. Probably, it is a new strategy to cure NSCLC with mutation T790M in EGFR by inhibiting the IL-6/JAK1/STAT3 signaling pathway. It has been reported that the JAK inhibitor pyridone 6 (P6) combined with afatinib can inhibit the growth of NSCLC cells in vitro and in vivo (10). TG101348, a kind of JAK2 inhibitor, also had the ability to overcome erlotinib-resistance in NSCLC cells with mutated EGFR (14).

More recently, it has been found that matrine, an extract of the Chinese traditional medicine radix Sophorae flavescentis, has antitumor activity in vitro and in vivo. Multiple studies have confirmed that matrine can inhibit the proliferation of leukemia cells by suppressing DNA synthesis and inducing apoptosis, and in combination with commonly used chemotherapeutics can effectively reverse the drug resistance of leukemia cells $(15,16)$. Matrine can also decrease the expression of IL-6 and activation of the IL-6 receptor-mediated JAK/STAT3 signaling pathway (17-19).

Whether matrine can inhibit activation of the JAK1/STAT3 signaling pathway in NSCLC is still unknown. In the present study, we investigated the effects of matrine treatment on the 
growth of H1975 cells and activation of the JAK1/STAT3 signaling pathway in the presence or absence of afatinib.

\section{Materials and methods}

Reagents. Anti-Bcl-2, anti-p-JAK1, anti-JAK1, anti-p-STAT3, anti-STAT3, anti- $\beta$-actin, anti-cleaved caspase- 3 , anti-caspase-3 and HRP-conjugated anti-rabbit antibodies and the IL-6 kit were purchased from Cell Signaling Technology, Inc., (Danvers, MA, USA). Matrine ( $\geq 99 \%$ purity by HPLC) was purchased from Sigma-Aldrich (St. Louis, MO, USA). Afatinib was purchased from SYNkinase (San Diego, CA, USA).

Cell culture. The T790M EGFR-mutant NCI-H1975 (H1975) cell line used in this study was obtained from the American Type Culture Collection (Manassas, VA, USA), and cultured in RPMI-1640, supplemented with $10 \%$ (v/v) fetal bovine serum (FBS) and 1\% (v/v) penicillin/streptomycin (all from Invitrogen, Waltham, MA, USA), and incubated at $37^{\circ} \mathrm{C}$ in a carbon dioxide incubator.

Cell viability assay. The effect of matrine on the growth of H1975 cells was detected with a Cell Counting Kit-8 (CCK-8). In the presence or absence of afatinib, $10^{5}$ cells per well in a 96-well plate were treated with or without matrine. Cell viability was detected after treatment with matrine or afatinib for $24 \mathrm{~h}$. Solution containing WST-8 [2-(2-methoxy-4-nitrophenyl)-3-(4-nitrophenyl)-5-(2,4-disulfophenyl)-2H-tetrazolium, monosodium salt] was added to cells according to the manufacturer's instructions and absorbance was detected at $450 \mathrm{~nm}$. All experiments were performed in triplicate.

RNA extraction and real-time reverse transcription PCR (RT-PCR). Total RNA was extracted from cells with RNAiso plus reagent (Takara Bio, Dalian, China) according to the manufacturer's instructions. RNA concentration was determined with a spectrophotometer. First-strand cDNA was synthesized using a Transcriptor First Strand cDNA Synthesis kit (Roche Applied Science, Mannheim, Germany). The amplification conditions were as follows: $50^{\circ} \mathrm{C}$ for $2 \mathrm{~min}$ and $95^{\circ} \mathrm{C}$ for $10 \mathrm{~min}$, followed by 40 cycles of $95^{\circ} \mathrm{C}$ for $10 \mathrm{sec}$, $58^{\circ} \mathrm{C}$ for $10 \mathrm{sec}$, and $72^{\circ} \mathrm{C}$ for $10 \mathrm{sec}$. The PCR reaction was performed using a 7500 Fast Real-Time quantitative PCR System (Applied Biosystems; Life Technologies, Foster City, CA, USA). The primer sequences for real-time PCR of IL-6 were 5'-CACTCACCTCTTCAGAACGAAT-3' and 5'-TTT GTACTCATCTGCACAGCTC-3', and the primer sequences for the internal control were 5'-CCAGAG-CAAGAGAGGCAT CCT-3' and 5'-TAGATGGGCACAGTGTGGGTGA-3' (20). RNA levels were determined with the $2^{\Delta \Delta \mathrm{Ct}}$ method.

Apoptosis assay. Cell death was detected by flow cytometry with Annexin V-fluorescein isothiocyanate, according to the manufacturer's instructions (BD Biosciences, San Jose, CA, USA). Briefly, $1 \times 10^{6}$ cells were plated in six-well plates for $24 \mathrm{~h}$ followed by treatment with matrine or afatinib for $24 \mathrm{~h}$ prior to Annexin V and propidium iodide (PI) staining (FACSAria; BD Biosciences). For each dye, appropriate electronic compensation of the FACSAria sorter was performed to avoid overlapping of the two emission spectra.
Enzyme-linked immunosorbent asssay (ELISA). Supernatant was prepared from $\mathrm{H} 1975$ cells treated with or without matrine in the presence or absence of afatinib for $24 \mathrm{~h}$. The content of IL-6 from the supernatant was detected using IL-6 ELISA kit (Sigma-Aldrich).

Western blot analysis. Cells were dissolved in lysis buffer and the lysate was centrifuged at $10,000 \mathrm{xg}$ at $4^{\circ} \mathrm{C}$ for 30 min after incubation on ice for $50 \mathrm{~min}$. Lysate of equal concentration measured with the BCA kit was subjected to $12 \%$ (w/v) sodium dodecyl sulfate-polyacrylamide gel electrophoresis (SDS-PAGE) and transferred to polyvinylidene fluoride (PVDF) membrane (Amersham Hybond $^{\mathrm{TM}}$ P; GE Healthcare Bio-Sciences, Pittsburgh, PA, USA). After blocking in 5\% (w/v) skimmed milk or bovine serum albumin (BSA), membranes were incubated with anti-Bcl-2 (1:1,000), anti-p-JAK1 (1:1,000), anti-JAK1 (1:1,000), anti-p-STAT3 $(1: 1,000)$, anti-STAT3 $(1: 1,000)$, anti-cleaved caspase-3 $(1: 1,000)$, anti-caspase-3 $(1: 1,000)$, or anti- $\beta$-actin $(1: 2,000)$ antibodies, followed by incubation with the appropriate secondary antibody for $1 \mathrm{~h}$ at room temperature. Finally, proteins were detected by chemiluminescence (PerkinElmer Life and Analytical Sciences, Inc., Massachusetts, MA, USA).

Small interfering RNA (siRNA). H1975 cells were transfected with IL-6 siRNA (sc-39627; Santa Cruz Biotechnology, Inc., Paso Robles, CA, USA) or negative control siRNA (sc-37007; Santa Cruz Biotechnology, Inc.) using Lipofectamine 2000 (Invitrogen). Under subconfluent conditions, transfection reagents with 12 pmol of siRNA and $16 \mu \mathrm{l}$ of Lipofectamine 2000 in a final volume of $1.6 \mathrm{ml}$ with Opti-MEM I (Invitrogen) were added to each flask, and incubated for $48 \mathrm{~h}$ prior to treatment with matrine.

Tumor xenograft studies. H1975 cells were harvested and resuspended in serum-free media, and $5 \times 10^{6}$ cells were injected subcutaneously under the back skin of 5-to-6-week-old male $\mathrm{BALB} / \mathrm{c}$ nude mice. The mice were treated with or without matrine $(5 \mathrm{~g} / \mathrm{kg})$ in the presence or absence of afatinib $(5 \mathrm{mg} / \mathrm{kg}$ ) formulated in saline by intraperitoneal injection once daily for 4 weeks. Tumor volume was estimated once every 3 days for 4 weeks with the following formula: volume $=l \times w^{2} \times 0.536$, where $l$ and $w$ are perpendicular measured diameters. All treatments on animals were in accordance with the ethical standards.

Statistical analysis. Data are represented as means \pm standard error of the mean (SEM) of three independent experiments and analyzed using the Student's t-test. $\mathrm{P}<0.05$ was considered to indicate a statistically significant difference.

\section{Results}

Matrine inhibits the growth of H1975 cells by inducing apoptosis. H1975 cells are resistant to TKIs because of the T790M mutation in the EGFR. To explore the effects of matrine on the growth of H1975 cells, cell viability was assessed with the Cell Counting Kit-8 (CCK-8) assay. Our results indicated that matrine inhibits the growth of H1975 cells in a dose-dependent manner with an $\mathrm{IC}_{50}$ value of approximately $2 \mathrm{mM}$ (Fig. 1B). Cell viability 
A

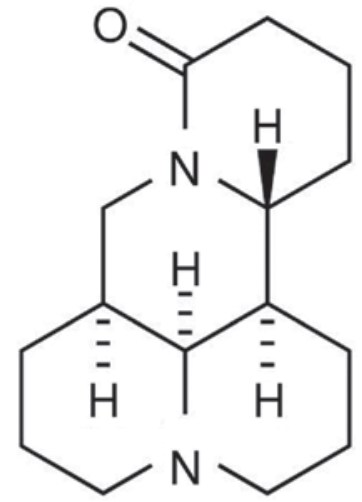

B

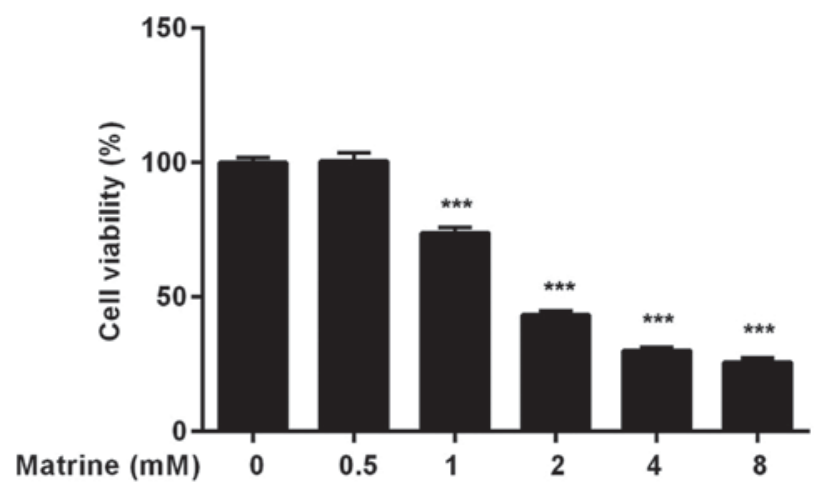

Figure 1. Effects of matrine treatment on viability of H1975 cells. (A) Structure of matrine. (B) Cell viability was measured using a Cell Counting Kit- 8 (CCK-8) assay and shown by the absorbance at $450 \mathrm{~nm}$ after treatment with various concentrations of matrine for $24 \mathrm{~h}$. Data are representative of three independent experiments and expressed as means \pm standard error of the mean (SEM). ${ }^{* * * *} \mathrm{P}<0.001$ compared with control.

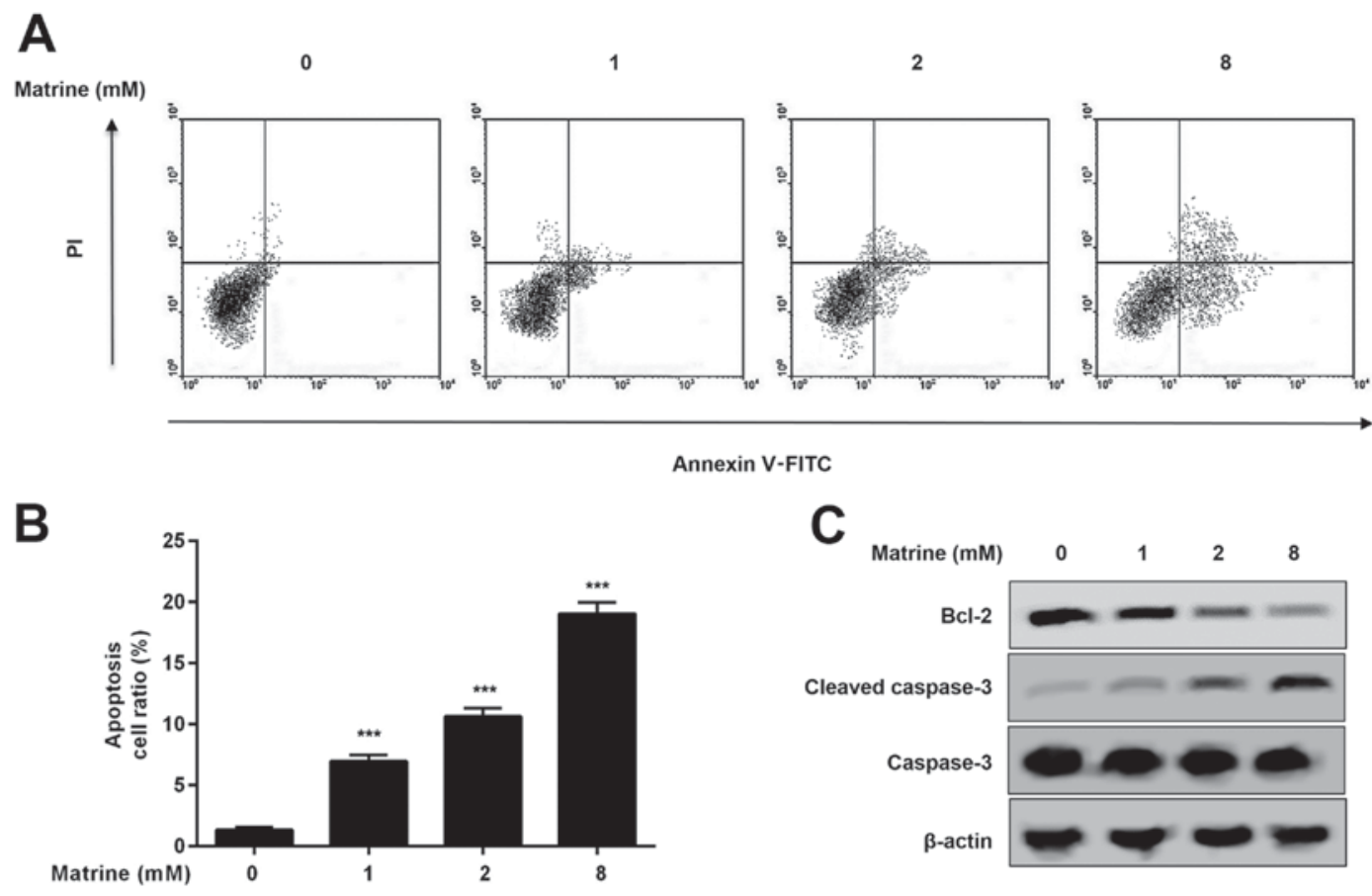

Figure 2. Effects of matrine treatment on apoptosis in H1975 cells. (A) Apoptosis was detected by Annexin V-FITC and propidium iodide (PI) staining in H1975 cells treated with matrine. (B) Graph of (A). (C) Protein levels of Bcl-2, cleaved caspase-3, and total caspase-3 were detected by western blot analysis in $\mathrm{H} 1975$ cells treated with various concentrations of matrine for $24 \mathrm{~h}$. $\beta$-actin is included as a loading control. Data are representative of three independent experiments and expressed as means \pm SEM. ${ }^{* * *} \mathrm{P}<0.001$ compared with control.

is dependent on cell proliferation and cell death. Here, matrine treatment induced apoptosis and cleavage of caspase-3 in H1975 cells (Fig. 2A-C). In addition, expression of the anti-apoptotic protein B-cell lymphoma 2 (Bcl-2) decreased in $\mathrm{H} 1975$ cells treated with matrine (Fig. 2C). Our results indicate that matrine inhibits growth of $\mathrm{H} 1975$ cells, likely due to the decrease to Bcl-2 expression and the resulting increase in apoptosis.

Matrine inhibits the IL-6/JAK1/STAT3 signaling pathway in H1975 cells. The JAK1/STAT3 signaling pathway can result in several biological reactions that prevent apoptosis. To explore whether matrine treatment impacts the JAK1/STAT3 signaling pathway in H1975 cells, we measured the phosphorylation of
JAK1 and STAT3 by western blot analysis following treatment with different concentrationsof matrine. Our results demonstrate that matrine decreased the levels of phosphoryalted JAK1 (p-JAK1) and phosphoryalted STAT3 (p-STAT3) in a dose-dependent manner, thus inhibiting the JAK1/STAT3 signaling pathway (Fig. 3A). The binding of IL-6 to its receptor, IL-6R, contributes to the phosphorylation of JAK1 and activation of the JAK1/STAT3 signaling pathway. To explore whether IL-6 is associated with inhibition of JAK1/STAT3 signaling, we determined IL-6 levels with real-time reverse transcription (RT-PCR) and an enzyme-linked immunosorbent assay (ELISA) in H1975 cells treated with various concentrations of matrine. We observed that matrine treatment results in a decrease in the 
A

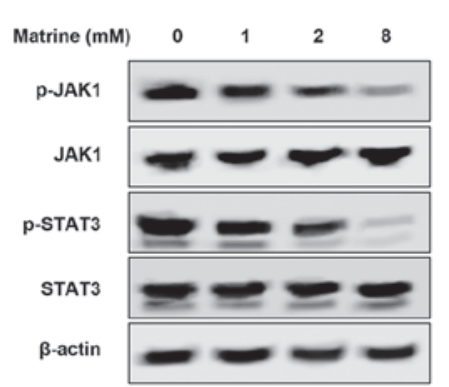

B

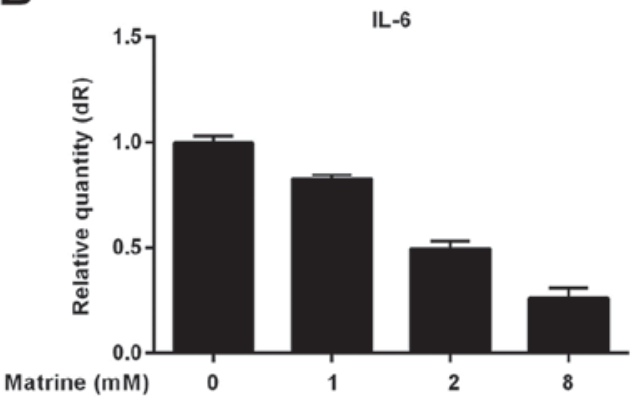

C

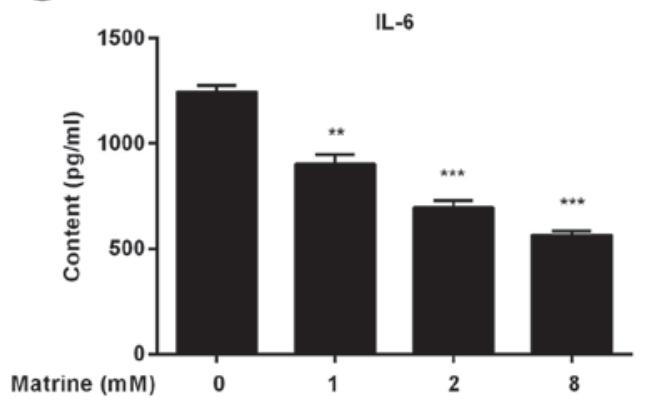

Figure 3. Effects of matrine treatment on the JAK1/STAT3 signaling pathway and IL-6 levels in H1975 cells. (A) Protein levels of p-JAK1 and p-STAT3 in H1975 cells treated with various concentrations of matrine for $24 \mathrm{~h}$ were detected by western blot analysis. (B) mRNA expression of IL-6 in H1975 cells treated with matrine for $24 \mathrm{~h}$ was detected using real-time RT-PCR. (C) Protein levels of IL-6 from the supernatant of H1975 cells treated with different concentrations of matrine were detected by enzyme-linked immunosorbent assay (ELISA). $\beta$-actin was included as an mRNA and protein loading control. Data are representative of three independent experiments and expressed as means \pm SEM. ${ }^{* *} \mathrm{P}<0.01$ and ${ }^{* * * *} \mathrm{P}<0.001$ compared with control.

A

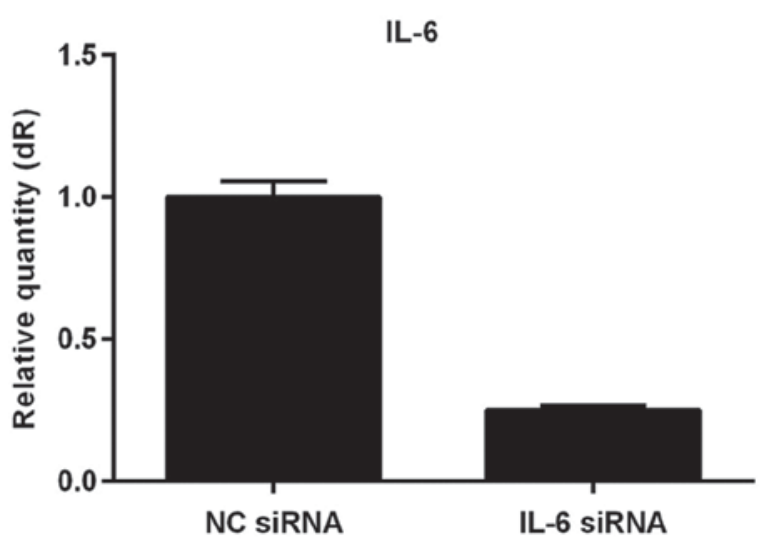

B

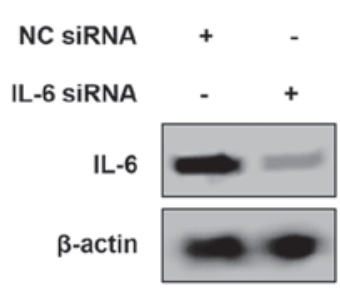

C

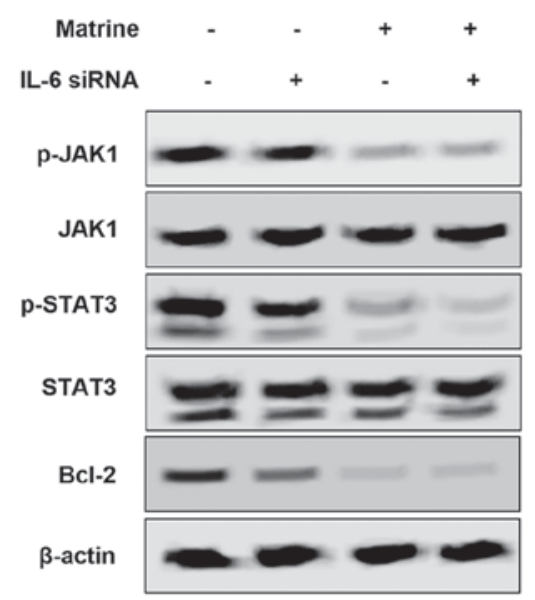

Figure 4. Effects of matrine treatment on the IL-6/JAK1/STAT3 signalign pathway in H1975 cells treated with IL-6 siRNA. (A) Transcriptional expression of IL-6 in H1975 cells treated with or without IL-6 siRNA was detected by real-time RT-PCR. (B) Protein levels of IL-6 from the supernatant of H1975 cells treated with or without IL-6 siRNA were detected by ELISA. (C) Protein levels of p-STAT3 and Bcl-2 in H1975 cells treated with or without IL-6 siRNA in the presence or absence of $2 \mathrm{mM}$ matrine were detected by western blot. $\beta$-actin was included as an mRNA and protein loading control. Data are representative of three independent experiments and expressed as means \pm SEM.

mRNA and protein levels of IL-6 in a dose-dependent manner (Fig. 3B and C).

Matrine induces apoptosis by regulating the IL-6/JAK1/STAT3 signaling pathway in H1975 cells. To investigate whether matrine treatment inhibits the growth of H1975 cells via the IL-6/JAK1/STAT3 signaling pathway, we targeted IL-6 with siRNA to evaluate the role of IL-6/JAK1/STAT3 signaling in matrine-induced apoptosis by real-time RT-PCR. Our results demonstrated that treatment with siRNA targeting IL-6, which markedly reduces the levles of IL-6 mRNA and protein (Fig. 4A and B), influenced the phosphorylation of JAK1 and STAT3 and the expression of Bcl-2, ultimately inhibiting cell growth (Figs. 4C and 5). To explore whether IL-6 is involved in matrine-induced apoptosis through the JAK1/STAT3 signaling pathway in H1975 cells, we also assessed molecular signal, apoptosis and cell viability in H1975 cells treated with or without IL-6 siRNA in the presence or absence of matrine. Our data demonstrated that IL-6
siRNA could not enhance inactivation of the JAK1/STAT3 signaling pathway and apoptosis in $\mathrm{H} 1975$ cells treated with matrine (Figs. 4C and 5A and B). Therefore, matrine treatment inhibits growth of H1975 cells via the IL-6/JAK1/STAT3 signaling pathway, decreases the expression of $\mathrm{Bcl}-2$, and induces apoptosis.

Combined treatment with afatinib and matrine has a greater effect on H1975 cells. We have seen that afatinib treatment not only decreases the viability of H1975 cells, but also induces the expression of IL- 6 and activation of the JAK1/STAT3 signaling pathway (Fig. 6A-C). Activation of the IL-6/JAK1/STAT3 signaling pathway likely increases the resistance of $\mathrm{H} 1975$ cells to afatinib. To investigate whether matrine can inhibit activation of the IL-6/JAK1/STAT3 signaling pathway stimulated by afatinib $\left(\mathrm{IC}_{50}\right.$ value of $\left.1 \mu \mathrm{M}\right)$ in $\mathrm{H} 1975$ cells, the expression of IL- 6 and phosphorylation of JAK1 and STAT3 were detected by western blot and ELISA in H1975 cells treated with matrine and afatinib. 
A
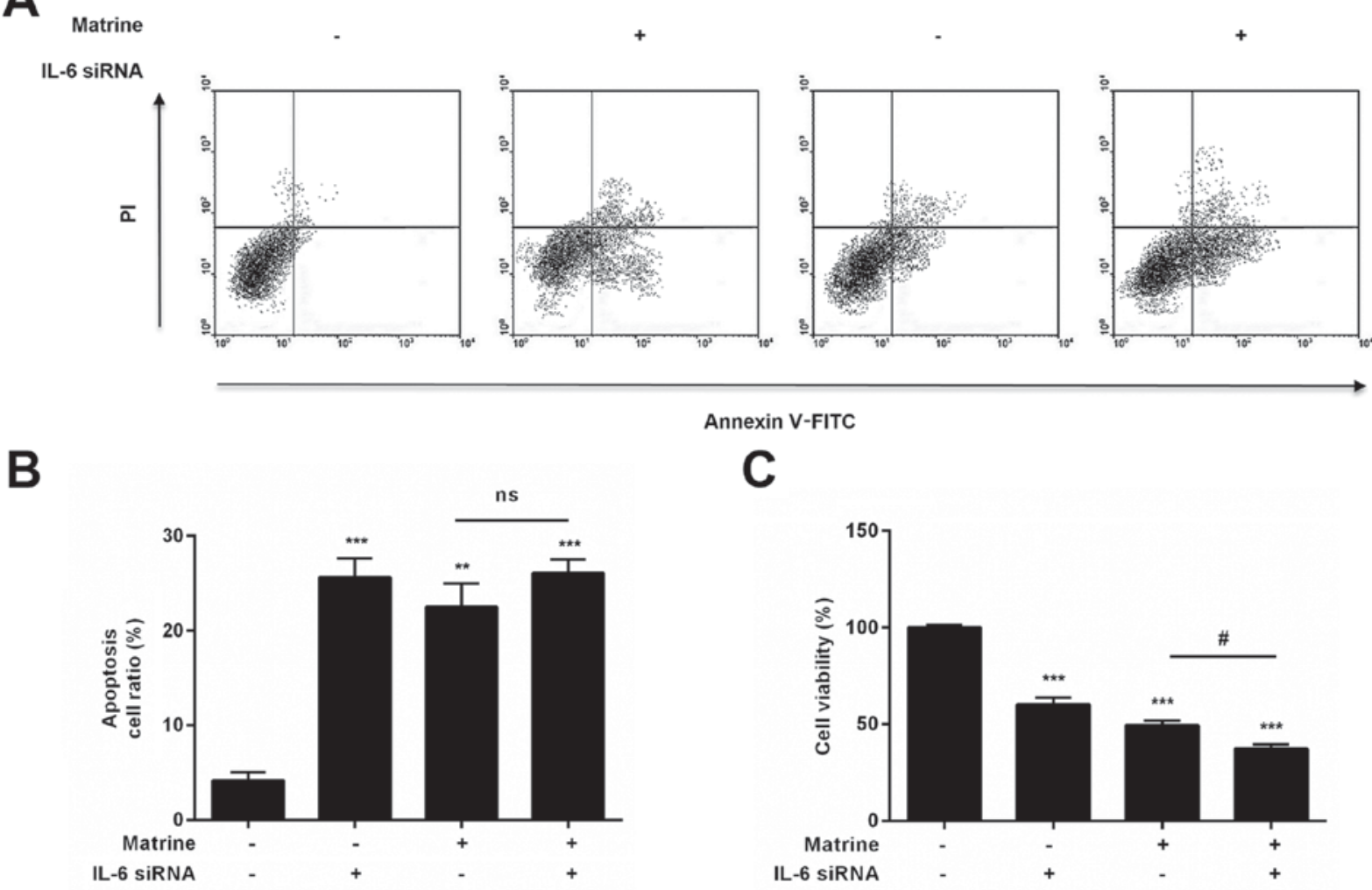

Annexin V-FITC

C

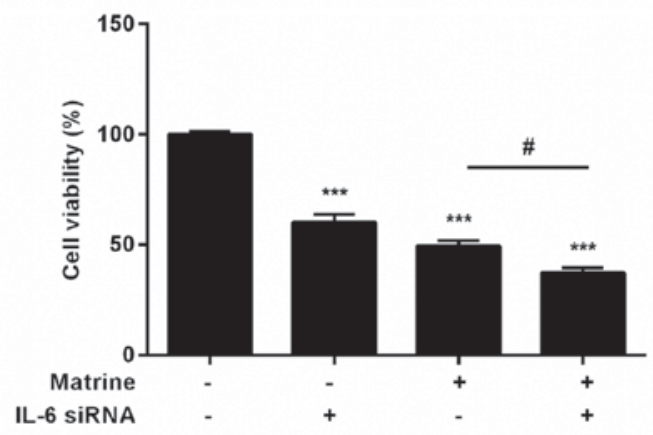

Figure 5. Effects of matrine treatment on apoptosis and viability of H1975 cells treated with IL-6 siRNA. (A) Apoptosis was determined by Annexin V-FITC and PI staining in H1975 cells treated with 2 mM matrine or IL-6 siRNA. (B) Graph of (A). (C) The inhibitory effects of IL-6 siRNA on the growth of H1975 cells treated with or without $2 \mathrm{mM}$ matrine were measured with the CCK- 8 assay and shown by the absorbance at $450 \mathrm{~nm}$. Data are representative of three independent experiments and expressed as means \pm SEM. ${ }^{* *} \mathrm{P}<0.01$ and ${ }^{* * *} \mathrm{P}<0.001$ compared with control. ${ }^{*} \mathrm{P}<0.05$ compared with the group treated with matrine in the absence of IL-6 siRNA.
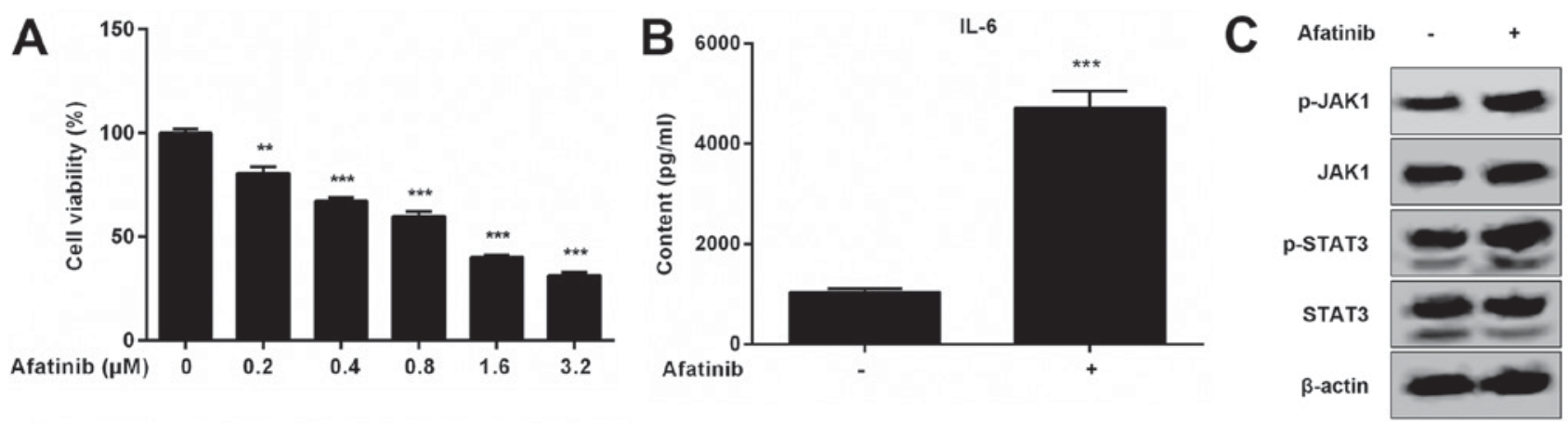

Figure 6. Effects of afatinib treatment on apoptosis and the IL-6/JAK1/STAT3 signaling pathway in H1975 cells. (A) Cell viability was measured with the CCK-8 assay and shown by the absorbance at $450 \mathrm{~nm}$ following treatment with various concentrations of afatinib for $24 \mathrm{~h}$. (B) Protein levels of IL-6 from the supernatant of $\mathrm{H} 1975$ cells treated with or without $1 \mu \mathrm{M}$ afatinib were detected by ELISA. (C) Activation of the JAK1/STAT3 signaling pathway was detected in $\mathrm{H} 1975$ cells in the presence or absence of $1 \mu \mathrm{M}$ afatinib by western blot. $\beta$-actin was included as an mRNA and protein loading control. Data are representative of three independent experiments and expressed as means \pm SEM. ${ }^{* *} \mathrm{P}<0.01$, and ${ }^{* * *} \mathrm{P}<0.001$ compared with control.

Our results demonstrated that matrine can inhibit activation of the IL-6/JAK1/STAT3 signaling pathway induced by afatinib and can also increase the inhibitory effects of afatinib on $\mathrm{H} 1975$ cells resulting from the increased rate of apoptosis (Fig. 7A-C). To explore whether matrine can also inhibit the growth of H1975 cells in vivo, we performed tumor xenograft studies. Treatment with afatinib and matrine decreased the tumor volume more dramatically than matrine or afatinib alone (Fig. 8). Therefore, matrine increased the toxicity of afatinib by regulating the IL-6/JAK1/STAT3 signaling pathway, and by decreasing the expression of Bcl-2 and the resistance of $\mathrm{H} 1975$ cells to afatinib.

\section{Discussion}

Mutations occur in NSCLC cells and result in resistance to EGFR inhibitors (TKIs), such as EGFR C797S mutation happened in NSCLC, which was resistant to AZD9291 (21). These mutations, 

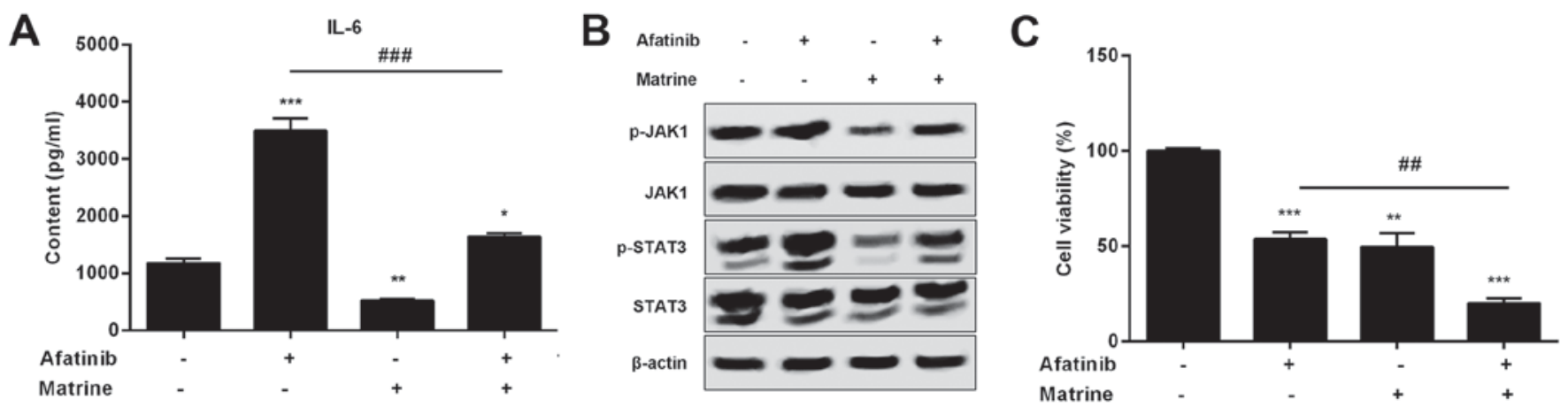

Figure 7. Effects of matrine and afatinib treatment on the growth of H1975 cells in vitro. (A) Protein levels of IL-6 from the supernatant of H1975 cells treated with or without $1 \mu \mathrm{M}$ afatinib in the presence or absence of $2 \mathrm{mM}$ matrine were detected by ELISA. (B) Protein levels of p-JAK1 and p-STAT3 were detected by western blot prepared from $\mathrm{H} 1975$ cells incubated with $1 \mu \mathrm{M}$ afatinib combined with $2 \mathrm{mM}$ matrine. (C) Effects of of afatinib and matrine treatment on the growth of H1975 cells were measured with the CCK-8 assay and shown by the absorbance at $450 \mathrm{~nm}$. $\beta$-actin was included as an mRNA and protein loading control. Data are representative of three independent experiments and expressed as means \pm SEM. ${ }^{*} \mathrm{P}<0.05,{ }^{* * *} \mathrm{P}<0.01$, and ${ }^{* * * *} \mathrm{P}<0.001$ compared with control. ${ }^{\# \#} \mathrm{P}<0.01$ compared with the group treated with afatinib in the absence of matrine.

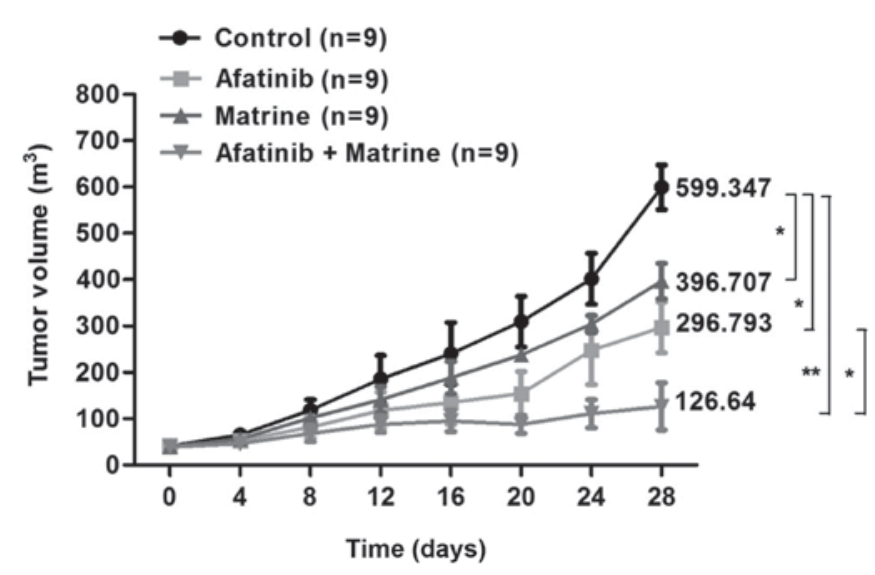

Figure 8. Effects of matrine and afatinib treatment on the growth of H1975 cells in vivo. After $5 \times 10^{6}$ cells were injected subcutaneously under the back skin of 5-to-6-week-old male BALB/c nude mice, mice were treated with or without matrine $(5 \mathrm{~g} / \mathrm{kg})$ in the presence or absence of afatinib $(5 \mathrm{mg} / \mathrm{kg})$ formulated in saline by intraperitoneal injection once daily for 4 weeks and tumor volume was estimated once every three days with the formula: volume $=l \times w^{2} \times 0.536$, where $l$ and $w$ are perpendicular measured diameters Data are expressed as means \pm SEM. ${ }^{*} \mathrm{P}<0.05,{ }^{* *} \mathrm{P}<0.01$.

especially the T790 M mutation, are a threat to patients with NSCLC $(7,8)$. It is important to overcome EGFR (T790M) resistance in NSCLC (22). Consequently, understanding the mechanism by which the mutated EGFR acquires resistance to TKI treatment will be important for the development of new therapies to cure NSCLC or prevent further progression of the disease. It has been suggested that the T790M mutation changes the structure of the EGFR, inhibiting its ability to bind TKIs, thus decreasing the inhibitory effects of TKIs on EGFR activation (8). It has been shown recently that TKIs can activate several signaling pathways, including the JAK1/STAT3 pathway, by inducing expression of IL-6, which results in a decline to TKI toxicity and an increase in the survival of NSCLC cells with the T790M mutation (9-11). The IL-6/JAK1/STAT3 signaling pathway, stimulated by TKIs such as afatinib, can also promote the EMT, thereby increasing cancer cell migration, invasion, and ultimately metastasis $(12,13)$. Inhibiting the expression of IL-6 or inactivating the JAK1/STAT3 signaling pathway has been reported to increase the toxicity of TKIs toward TKI-resistant NSCLC cells $(10,14,23)$. Targeting the IL-6/JAK1/STAT3 signaling pathway will be an important strategy for the treatment of patients with TKI-resistant NSCLC.

Matrine is an alkaloid prepared from the leguminous plant radix Sophorae flavescentis. It plays an important role in the regulation of IL-6 expression and activation of the JAK1/STAT3 signaling pathway (17-19,24). A number of previous studies have reported the effects of matrine on NSCLC cells with the T790M mutation, including $\mathrm{H} 1975$ cells, in the presence or absence of a TKI. However, there remain some questions as to whether matrine can inhibit the expression of IL-6 or promote inactivation of the JAK1/STAT3 signaling pathway in H1975 cells stimulated by afatinib, and whether a combination of matrine and afatinib can further inhibit H1975 cells.

In the present study, we determined the effect of matrine treatment on the growth of $\mathrm{H} 1975$ cells treated without afatinib with the CCK- 8 assay. Our results demonstrated that matrine reduces the viability of $\mathrm{H} 1975$ cells, likely due to the decreased expression of $\mathrm{Bcl}-2$ and increased rate of apoptosis. Activation of the JAK1/STAT3 signaling pathway was detected by western blot and we found that matrine treatment inhibited phosphorylation of JAK1 and STAT3 in a dose-dependent manner.

IL- 6 participates in activation of the JAK1/STAT3 signaling pathway by binding the IL- 6 receptor complexed with glycoprotein 130 (gp130). Our results indicated that matrine treatment decreased IL- 6 protein levels as a result of its dose-dependent inhibition of IL-6 transcription. To identify inactivation of the JAK1/STAT3 signaling pathway as a result of IL-6 inhibition and the role of the IL-6/JAK1/STAT3 pathway in matrine-induced apoptosis in H1975 cells, we targeted IL-6 with siRNA. Down regulation of IL-6 by siRNA inhibited phosphorylation of JAK1 and STAT3. However, IL-6 siRNA could not enhance inactivation of the JAK1/STAT3 signaling pathway, inhibition of Bcl-2 expression, or apoptosis in H1975 cells treated with matrine. In summary, matrine inhibits the IL-6/JAK1/STAT3 signaling pathway and decreases Bcl-2 expression, causing an increased rate of apoptosis and a decline in the viability of $\mathrm{H} 1975$ cells.

We then studied whether matrine treatment significantly inhibited H1975 cells stimulated by afatinib. Our results 
indicated that treatment with both matrine and afatinib can trigger apoptosis of $\mathrm{H} 1975$ cells and that matrine treatment can inhibit the IL-6/JAK1/STAT3 pathway induced by afatinib, thus increasing the inhibitory effects of afatinib. In addition, treatment with both matrine and afatinib had a more dramatic inhibitory effect on mouse tumor volume in vivo.

We found that matrine could enhance the toxicity of afatinib toward H1975 cells harboring the T790M mutation by suppressing activation of the IL-6/JAK1/STAT3 signaling pathway induced by afatinib. Treatment with both matrine and afatinib will likely be successful for patients of NSCLC with the T790M mutation.

\section{Acknowledgements}

The present study was supported by the Science and Technology Development Fund Projects of The First Affiliated Hospital, College of Medicine, Zhejiang University (grant no. B1519). Experimental animals were provided by the Experimental Animal Center of the Zhejiang Academy of Medical Sciences. We thank Professor Jian-Ying Zhou for the experimental design.

\section{References}

1. Herbst RS, Heymach JV and Lippman SM: Lung cancer. N Engl J Med 359: 1367-1380, 2008.

2. Spira A and Ettinger DS: Multidisciplinary management of lung cancer. N Engl J Med 350: 379-392, 2004.

3. Lanaya H, Natarajan A, Komposch K, Li L, Amberg N, Chen L, Wculek SK, Hammer M, Zenz R, Peck-Radosavljevic M, et al: EGFR has a tumour-promoting role in liver macrophages during hepatocellular carcinoma formation. Nat Cell Biol 16: 972-981, 2014.

4. Pao W and Chmielecki J: Rational, biologically based treatment of EGFR-mutant non-small-cell lung cancer. Nat Rev Cancer 10: 760-774, 2010.

5. Maemondo M, Inoue A, Kobayashi K, Sugawara S, Oizumi S, Isobe H, Gemma A, Harada M, Yoshizawa H, Kinoshita I, et al: Gefitinib or chemotherapy for non-small-cell lung cancer with mutated EGFR. N Engl J Med 362: 2380-2388, 2010.

6. Pirker R, Pereira JR, von Pawel J, Krzakowski M, Ramlau R, Park K, de Marinis F, Eberhardt WE, Paz-Ares L, Störkel S, et al: EGFR expression as a predictor of survival for first-line chemotherapy plus cetuximab in patients with advanced non-small-cell lung cancer: Analysis of data from the phase 3 FLEX study. Lancet Oncol 13: 33-42, 2012.

7. Rosell R, Carcereny E, Gervais R, Vergnenegre A, Massuti B, Felip E, Palmero R, Garcia-Gomez R, Pallares C, Sanchez JM, et al: Erlotinib versus standard chemotherapy as first-line treatment for European patients with advanced EGFR mutation-positive non-small-cell lung cancer (EURTAC): A multicentre, open-label, randomised phase 3 trial. Lancet Oncol 13: 239-246, 2012.

8. Kobayashi S, Boggon TJ, Dayaram T, Jänne PA, Kocher O, Meyerson M, Johnson BE, Eck MJ, Tenen DG and Halmos B: EGFR mutation and resistance of non-small-cell lung cancer to gefitinib. N Engl J Med 352: 786-792, 2005.

9. Gao SP, Mark KG, Leslie K, Pao W, Motoi N, Gerald WL, Travis WD, Bornmann W, Veach D, Clarkson B and Bromberg JF: Mutations in the EGFR kinase domain mediate STAT3 activation via IL-6 production in human lung adenocarcinomas. J Clin Invest 117: 3846-3856, 2007.
10. Kim SM, Kwon OJ, Hong YK, Kim JH, Solca F, Ha SJ, Soo RA, Christensen JG, Lee JH and Cho BC: Activation of IL-6R/JAK1/STAT3 signaling induces de novo resistance to irreversible EGFR inhibitors in non-small cell lung cancer with T790M resistance mutation. Mol Cancer Ther 11: 2254-2264, 2012.

11. Li J, Lan T, Zhang C, Zeng C, Hou J, Yang Z, Zhang M, Liu J and Liu B: Reciprocal activation between IL-6/STAT3 and NOX4/Akt signaling promotes proliferation and survival of non-small cell lung cancer cells. Oncotarget 6: 1031-1048, 2015.

12. Abulaiti A, Shintani Y, Funaki S, Nakagiri T, Inoue M, Sawabata N, Minami M and Okumura M: Interaction between non-small-cell lung cancer cells and fibroblasts via enhancement of TGF- $\beta 1$ signaling by IL-6. Lung Cancer 82: 204-213, 2013.

13. Chen J, Lan T, Zhang W, Dong L, Kang N, Zhang S, Fu M, Liu B, Liu K and Zhan Q: Feed-forward reciprocal activation of PAFR and STAT3 regulates epithelial-mesenchymal transition in non-small cell lung cancer. Cancer Res 75: 4198-4210, 2015.

14. Zhang FQ, Yang WT, Duan SZ, Xia YC, Zhu RY and Chen YB: JAK2 inhibitor TG101348 overcomes erlotinib-resistance in non-small cell lung carcinoma cells with mutated EGF receptor. Oncotarget 6: 14329-14343, 2015.

15. Liu Y, Xu Y, Ji W, Li X, Sun B, Gao Q and Su C: Anti-tumor activities of matrine and oxymatrine: Literature review. Tumour Biol 35: 5111-5119, 2014.

16. Zhang LP, Jiang JK, Tam JW, Zhang Y, Liu XS, Xu XR, Liu BZ and $\mathrm{He}$ YJ: Effects of matrine on proliferation and differentiation in K-562 cells. Leuk Res 25: 793-800, 2001.

17. Lu X, Zhu Z, Jiang L, Sun X, Jia Z, Qian S, Li J and Ma L: Matrine increases NKG2D ligand ULBP2 in K562 cells via inhibiting JAK/STAT3 pathway: A potential mechanism underlying the immunotherapy of matrine in leukemia. Am J Transl Res 7: 1838-1849, 2015.

18. Lin W, Zhang JP, Hu ZL and Qian DH: Inhibitory effect of matrine on lipopolysacchride-induced tumor necrosis factor and interleukin-6 production from rat Kupffer cells. Yao Xue Xue Bao 32: 93-96, 1997 (In Chinese).

19. Zhang Y, Wang S, Li Y, Xiao Z, Hu Z and Zhang J: Sophocarpine and matrine inhibit the production of TNF-alpha and IL-6 in murine macrophages and prevent cachexia-related symptoms induced by colon 26 adenocarcinoma in mice. Int Immunopharmacol 8: 1767-1772, 2008.

20. Liu X, Ye F, Xiong H, Hu DN, Limb GA, Xie T, Peng L, Zhang P, Wei Y, Zhang W, et al: IL-1 $\beta$ induces IL- 6 production in retinal Müller cells predominantly through the activation of P38 MAPK/NF- $\kappa$ B signaling pathway. Exp Cell Res 331: 223-231, 2015.

21. Thress KS, Paweletz CP, Felip E, Cho BC, Stetson D, Dougherty B, Lai Z, Markovets A, Vivancos A, Kuang Y, et al: Acquired EGFR C797S mutation mediates resistance to AZD9291 in non-small cell lung cancer harboring EGFR T790M. Nat Med 21: 560-562, 2015.

22. Jia Y, Yun CH, Park E, Ercan D, Manuia M, Juarez J, Xu C, Rhee K, Chen T, Zhang H, et al: Overcoming EGFR(T790M) and EGFR(C797S) resistance with mutant-selective allosteric inhibitors. Nature 534: 129-132, 2016.

23. Nan J, Du Y, Chen X, Bai Q, Wang Y, Zhang X, Zhu N, Zhang J, Hou J, Wang Q and Yang J: TPCA-1 is a direct dual inhibitor of STAT3 and NF- $\kappa$ B and regresses mutant EGFR-associated human non-small cell lung cancers. Mol Cancer Ther 13: 617-629, 2014.

24. Ma L, Zhu Z, Jiang L, Sun X, Lu X, Zhou M, Qian S and Jianyong L: Matrine suppresses cell growth of human chronic myeloid leukemia cells via its inhibition of the interleukin-6/Janus activated kinase/signal transducer and activator of transcription 3 signaling cohort. Leuk Lymphoma 56: 2923-2930, 2015. 